\title{
Study on Antioxidant Activity of Peanut Meal using Bacillus Natto by Solid State Fermentation
}

\section{WANG Yanping ${ }^{1}$, HU Yingfen² , MA Yuping ${ }^{3}$, ZHANG Yuanjie ${ }^{3}$, LIU}

\section{Qing 3}

Qingdao University, Qingdao, 266071

Foundation items: Project supposed by science and technology development planning project of Shandong province (2014GSF20011)

Abstract: This experiment carried out a preliminary study on solid state fermentation of Bacillus natto peanut meal. Determination of the scavenging activities to hydroxyl free radical scavenging rate to determine its antioxidant activity. The optimal fermentation conditions were obtained by single factor and response surface methodology as follows: $38.8 \mathrm{~h}$ of Fermentation time, $37^{\circ} \mathrm{C}$ of fermentation temperature, material liquid ratiol:0.44, $6.48 \%$ of inoculation amount. Under this condition, the hydroxy free radical, iron reduction capacity and DPPH radical scavenging rate were $87.3 \% 、 0.36$ (OD value) and $73.4 \%$, respectively.

Keywords: Peanut Meal; Bacillus Natto; Response Surface Methodology; Antioxidant Activity

\section{Introduction}

Peanut is one of the five major oil-bearing crops in the world. The protein content of peanut is $25 \%-30 \%$ and it contains 8 necessary amino acid of personal body ${ }^{[1]}$.In the markets, the peanut protein product is primarily peanut protein powder, which is used as the main raw material during food processing ${ }^{[2]}$.Peanut meal is a by-product of the process of making peanut oil, which is used mainly for feed.However, peanut meal is rich in protein and there are better ways to put this protein resource to use. Utilizing the solid state fermentation to prepare antioxidant peptides would not only make full use of this resource but also enlarge the scope of finely and deeply processed peanuts, which present significant economic and social benefits.

Bacillus subtilis is an important starter culture for

\section{fermented}

soybean foods like Japanese natto, Thai thuanao,Indian kinema, and Chinese douchi have reported the antioxidant activity and safety of red bean fermented by B. subtilis IMR-NK $1^{[3-4]}$. In addition, the characteristics of the starter B. subtilis also play an important role in the properties and functionality of the fermented product ${ }^{[5]}$.

In the present study, the solid state fermentation technology was evaluated and the operational parameters were optimized using single factor-experiments and a response surface methodology (RSM) experimental design in order to increase its antioxidant activities. At the same time, antioxidant activities of the peanut meal fermented liquid were determined. The objective of this work is to 
provide a theoretical basis for the preparing peanut meal fermented liquid in order to improve its antioxidant activities.

\section{Materials and methods}

Materials peanut meal was from Qingdao Jiali Group Co.,Ltd.bean pulp and wheat bran were purchased from local market. Bacillus subtilis was isolated and conserved in this laboratory. $\mathrm{H}_{2} \mathrm{O}_{2}, \mathrm{FeSO}_{4}, \mathrm{C}_{6} \mathrm{H}_{4}(\mathrm{OH}) \mathrm{COOH}$,trichloroaceti c acid (TCA), $\mathrm{FeCl}_{3}, \mathrm{~K}_{3} \mathrm{Fe}(\mathrm{CN})_{6}, \mathrm{FeCl}_{2}$ were analytical reagents produced by Sinopharm Chemical Reagent Co., Ltd. China. 1, 1 - Diphenyl- 2 - picrylhydrazyl (DPPH) was purchased from Sigma.

Technology of bacillus subtilis solid state fermentation. Bacillus subtilis seed and sterile water were added to solid state fermentation medium(30g) and cultured at a certain temperature for a certain time. When fermentation was over and sterile $0.9 \%$ NaClwas added to fermentation system. The fermentation product was oscillated at constant temperature water bath for a certain time. After centrifugation the supernatant was to be analyzed.
Single factor experiment design. The factors and levels of single-factor experiments are Fermentation time of $24,30,36,25,42, \quad 48 \mathrm{~h}$,fermentation temperatureof $29,33,37,41,45^{\circ} \mathrm{C}$,material liquid ratio of 1:0.3, 1:0.4, 1:0.5, 1:0.6, 1:0.7, inoculation amount of 2.2,4.5,6.8,9.1,11.4\%, respectively. The basic fermentation conditions of single factor experiment are $24 \mathrm{~h}$ of Fermentation time, the ratio of the substrate for peanut meal, soybean meal, bran $=7: 2: 1,37{ }^{\circ} \mathrm{C}$ of fermentation temperature, Moisture ratio1:0.4, 6.8\% of inoculation amount.

Box-Behnken Design (BBD). According to the result of single factor experiment, the fermentation temperature remains unchanged. By using Box-Behnken Design (BBD)the three factors including Time(A),Moisture ratio(B), Inoculum size(C), which had significant influence on the hydroxyl free radical scavenging rate were chosen to conduct three factors and three levels response surface methodology(RSM) experiment. The hydroxyl free radical scavenging $\operatorname{rate}(Y)$ was as response variables. The design of experimental factors and codes was mentioned in Table1. The experiment plan was designed and itsresult was analyzed using Design Expert Software (Static Made Easy, Minneapolis, MN, USA.Version 8.0.6).

Table 1 Design of experimental factors and codes

\begin{tabular}{cccccc}
\hline & & \multicolumn{5}{c}{ Range and level } \\
\cline { 3 - 6 } Process variables & Codes & -1 & 0 & 1 \\
\hline Time[h] & $\mathrm{A}$ & 30 & 36 & 42 \\
solid-to-liquid ratio & $\mathrm{B}$ & $1: 0.3$ & $1: 0.4$ & $1: 0.5$ \\
Inoculum size[\%] & $\mathrm{C}$ & 4.5 & 6.8 & 9.1 \\
\hline
\end{tabular}

\section{Hydroxyl Free Radical Scavenging Activity}

Hydroxyl free radical scavenging activity was determined according to the method of Amarowiczet al., ${ }^{[6]} .2 \mathrm{~mL}$ of sample solution, $2 \mathrm{~mL}$ of sample solution, and $2 \mathrm{~mL}$ of distilled water were placed in three test tubes, respectively. Then $2 \mathrm{~mL}$ of $\mathrm{FeSO}_{4}(6$ $\mathrm{mmol} / \mathrm{L})$ and $\mathrm{H}_{2} \mathrm{O}_{2}(6 \mathrm{mmol} / \mathrm{L})$ were added to above three tubes, respectively and mixed vigorously. After standing for $10 \mathrm{~min}$ at roomtemperature, three $2 \mathrm{~mL}$ of solutions salicylic acid (6 mmol/L), distilled water and salicylic acid( $6 \mathrm{mmol} / \mathrm{L})$ were added to the above three tubes, respectively. After standing for $30 \mathrm{~min}$, the absorbances of the resulting solutions were recorded at $510 \mathrm{~nm}$ using a spectrophotometer. The absorption values of tubes 1,2 , and 3 were $\mathrm{Ai}, \mathrm{Aj}$, and $\mathrm{A} 0$, respectively. The hydroxyl free radical scavenging rate can be calculated as follows:

Hydroxyl free radical scavenging rate 
$(\%)=\left[1-\left(\mathrm{A}_{\mathrm{i}}-\mathrm{A}_{\mathrm{j}}\right) / \mathrm{A}_{0]} \times 100\right.$

\section{DPPH Free Radical Scavenging Activity}

DPPH free radical scavenging activity was determined according to the method of Khantaphant et al., ${ }^{[7]}$.

\section{Iron Reduction Capacity}

Iron reduction capacity was determined according to the method of You et al., ${ }^{[8]}$.

\section{Results and discussion}

\section{Effect of fermentation temperature on fermentation}

results. The effect of fermentation temperature on fermentation results is shown in Fig.1 (a). It was found that the higher the fermentation reaction temperature is, not the better is. Bacillus subtilis has suitable temperature range. Beyond a certain temperature, Bacillus subtilis become inactivity. In the range of $29^{\circ} \mathrm{C}-37^{\circ} \mathrm{C}$, the hydroxyl free radical scavenging rate presents the trend of increasing and then decreasing,which is $\max$ at $37^{\circ} \mathrm{C}$. Therefore, the temperature of $37^{\circ} \mathrm{C}$ acts as invariable factor of the response surface method experiment.

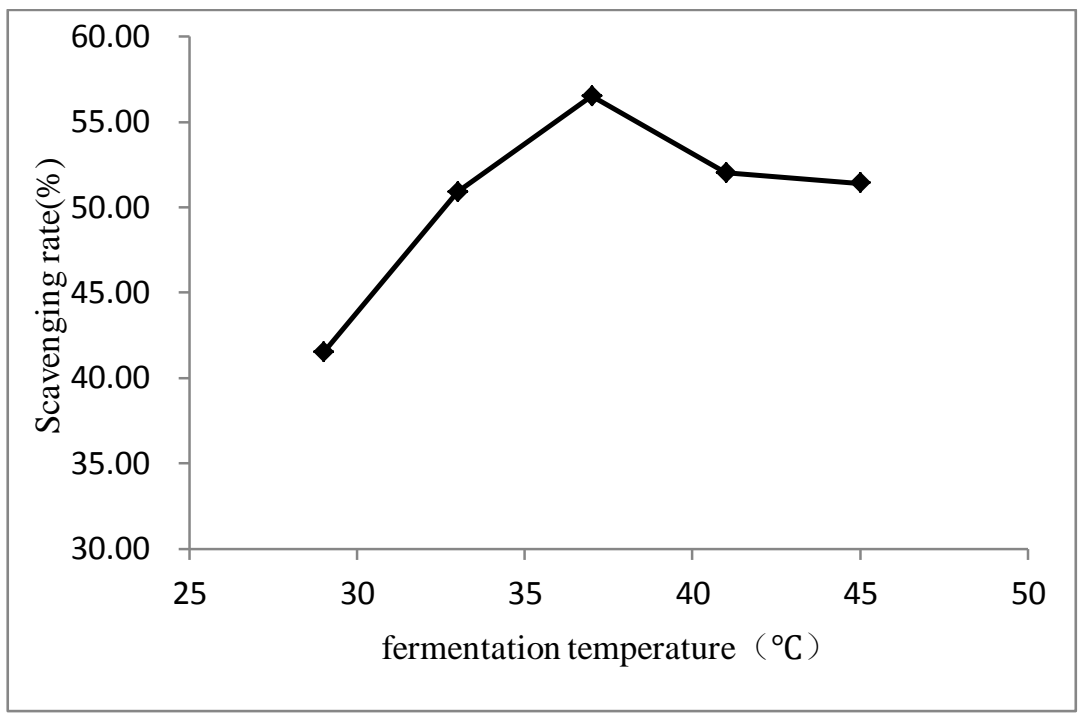

(a)

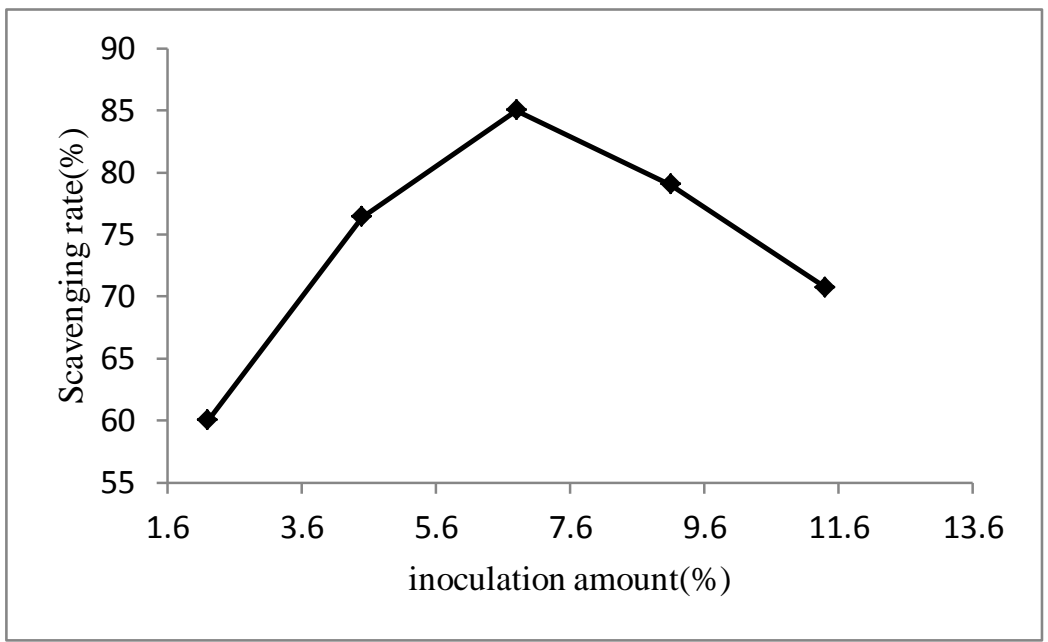

(b) 


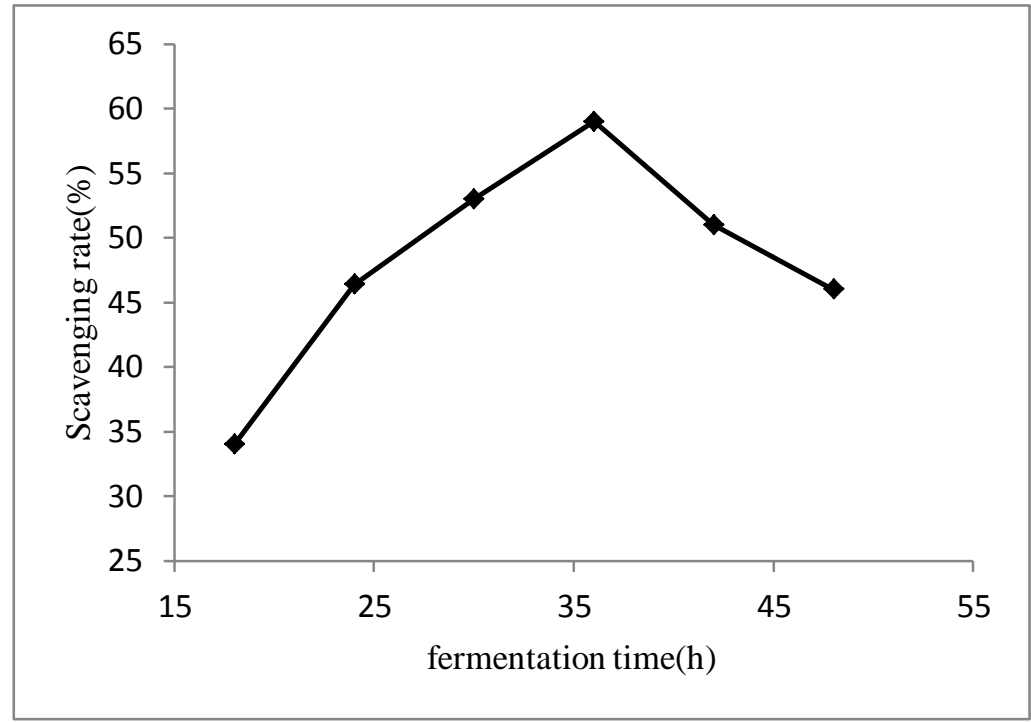

(c)

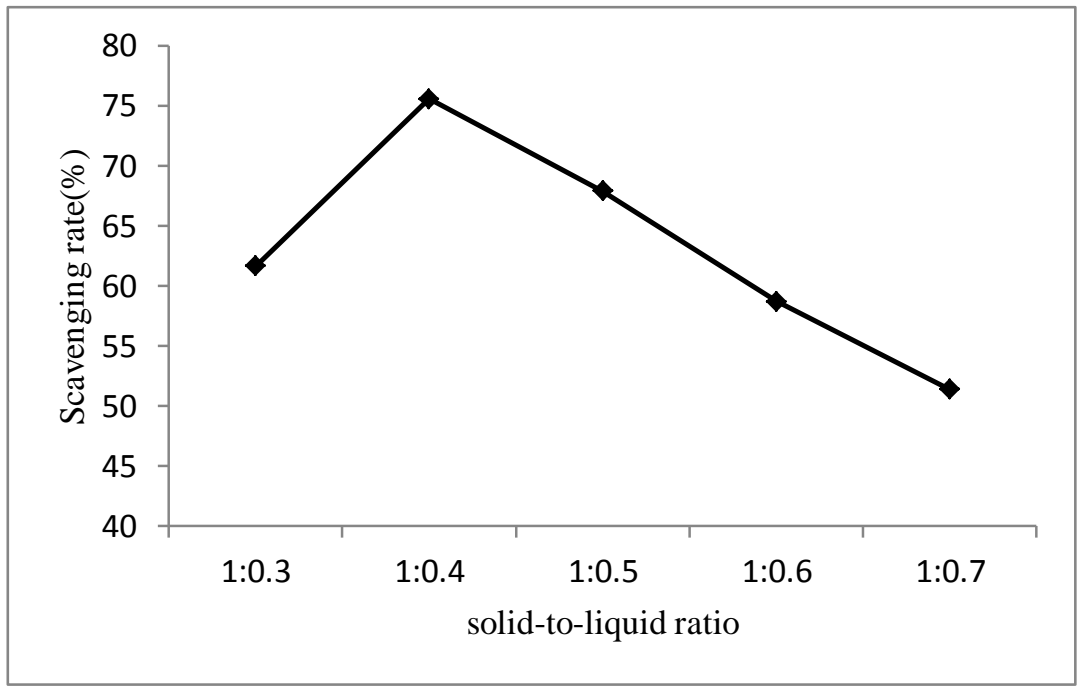

(d)

Fig.1 Effect of influencing factors, fermentation temperature (a), noculation amount(b), fermentation time(c), solid-to-liquid ratio(d) on fermentation results

Effect of inoculation amount on fermentation results. The effect of inoculation amount on fermentation results is shown in Fig.1 (b). The inoculation amount may be one of important factors responsible for the effect of results. The hydroxyl free radical scavenging rate increased rapidly when inoculation amount from $2.2 \%$ to6.8\% and then decreased .The result indicates that the maximum hydroxyl free radical scavenging rate was obtained at the substrate mass fraction of $6.8 \%$. Therefore, the inoculation amount range of $4.5-9.1 \%$ is chosen to be the response surface method experiment levels.

Effect of fermentation time on fermentation results. The effect of fermentation time on fermentation results is shown in Fig.1 (c). Fermentation time can also influence the effect of results. The fermentation product would be dependent on length of reaction time. When the fermentation time was $36 \mathrm{~h}$, the maximum antioxidant activity was obtained. Consequently, the hydrolysis time range of $30-42 \mathrm{~h}$ is chosen to be the response surface method experiment levels. 


\section{Effect of solid-to-liquid ratio on fermentation}

results. The effect of solid-to-liquid ratio on fermentation results is shown in Fig.1 (d). The hydroxyl free radical scavenging rate increased rapidly when solid-to-liquid ratio from 1:0.3to 1:0.4 and then decreased when solid-to-liquid ratio was up to1:0.4. The result indicates that the maximum hydroxyl free radical scavenging rate was obtained at the substrate mass fraction of 1:0.4.So, solid-to-liquid ratio 1:0.3-1:0.5 are used as response surface method experiment levels.

Table $2 \mathrm{BBD}$ and results

\begin{tabular}{|c|c|c|c|c|c|}
\hline Number & A & $\mathrm{B}$ & $\mathrm{C}$ & $\mathrm{Y}($ actual values $)(\%)$ & $\mathrm{Y}^{\prime}($ predicted values $)(\%)$ \\
\hline 1 & -1 & -1 & 0 & 57.80 & 59.65 \\
\hline 2 & 1 & -1 & 0 & 60.50 & 58.95 \\
\hline 3 & -1 & 1 & 0 & 48.10 & 49.65 \\
\hline 4 & 1 & 1 & 0 & 82.70 & 80.85 \\
\hline 5 & -1 & 0 & -1 & 38.60 & 34.73 \\
\hline 6 & 1 & 0 & -1 & 75.30 & 74.83 \\
\hline 7 & -1 & 0 & 1 & 65.70 & 66.18 \\
\hline 8 & 1 & 0 & 1 & 52.70 & 56.58 \\
\hline 9 & 0 & -1 & -1 & 53.60 & 55.63 \\
\hline 10 & 0 & 1 & -1 & 63.60 & 65.93 \\
\hline 11 & 0 & -1 & 1 & 68.90 & 66.58 \\
\hline 12 & 0 & 1 & 1 & 70.20 & 68.18 \\
\hline 13 & 0 & 0 & 0 & 81.00 & 84.10 \\
\hline 14 & 0 & 0 & 0 & 79.00 & 84.10 \\
\hline 15 & 0 & 0 & 0 & 85.30 & 84.10 \\
\hline 16 & 1 & 0 & -1 & 91.20 & 84.10 \\
\hline 17 & 0 & 0 & 0 & 84.00 & 84.10 \\
\hline
\end{tabular}

Design of RSM experiment and results. The solid state fermentation technology for producing antioxidant peptides was optimized by BBD of three factors and three levels, and the effects of these variables and their interactions, Time(A),Moisture ratio(B), Inoculum size(C) on the hydroxyl free radical scavenging rate of fermentation liquid were investigated. There are17 experimental points. Design and results see Table 2.
According to the data from experiment (Table 2), the regression analysis was carried out by using Design-Expert software, taking $\mathrm{Y}$ as the dependent variable, and each factor and their interactions as the independent variable. The regression equation was established as formula

$\mathrm{Y}=84.1+7.63 \mathrm{~A}+2.98 \mathrm{~B}+3.30 \mathrm{C}+7.97 \mathrm{AB}-12.43 \mathrm{AC}-2.18$

BC-13.91 $\mathrm{A}^{2}$

$-7.91 B^{2}-12.11 C^{2}$

The variance analysis results of this regression equation see Table3. The regression equation agrees well with the experimental data because the RSM model is statistically highly significant $(\mathrm{P}<0.01)$. As a result, the regression equation can analyze and predict actual experimental values. There are high relationships between the predicted and actual values and only about $4.19 \%$ data cannot be explained by this model due to the coefficient of correlation R 0.9581. Meanwhile, the insignificant Lack of fit $(\mathrm{P}>0.05)$ indicated that there are not abnormal values in experimental data. In addition, the smaller coefficient of variation also proved that the regression equation has good fitness. The effect of A on the hydroxyl free radical scavenging 
rateis at 0.01 significant levels and the effect of $\mathrm{A}^{2}$ 、 $\mathrm{B}^{2} 、 \mathrm{C}^{2}$ on the hydroxyl free radical scavenging rate were significant. The influencing sequence for hydroxyl free radical scavenging rate is $\mathrm{A}>C>B$, that

is, fermentation time $>$ Inoculum size $>$ solid-to-liquid ratio. The prediction about each experimental value is performed by using the regression equation and the results see Table 2.The model calculation and prediction show good agreement with the experimental results and it also accounts for the preferable fitness between the regression equation and actual experimental values. However, individual experiment error is great based on the influence of the instrumental error and the human factor in the course of the experiment. On the whole, the model performs well in experimental data.

Table 3 Variance analysis of RSM model for hydroxyl free radical scavenging rate

\begin{tabular}{|c|c|c|c|c|c|}
\hline Source & Sum of Squares & df & Mean Square & F Value & p-value $($ Prob $>F)$ \\
\hline Model & 3396.41 & 9 & 377.38 & 17.77 & 0.0005 \\
\hline A & 465.13 & 1 & 465.13 & 21.91 & 0.0023 \\
\hline B & 70.81 & 1 & 70.81 & 3.33 & 0.1106 \\
\hline $\mathrm{C}$ & 87.12 & 1 & 87.12 & 4.10 & 0.0824 \\
\hline $\mathrm{AB}$ & 254.40 & 1 & 254.40 & 11.98 & 0.0105 \\
\hline $\mathrm{AC}$ & 617.52 & 1 & 617.52 & 29.08 & 0.0010 \\
\hline $\mathrm{BC}$ & 18.92 & 1 & 18.92 & 0.89 & 0.3766 \\
\hline$A^{2}$ & 814.98 & 1 & 814.98 & 38.38 & 0.0004 \\
\hline $\mathrm{B}^{2}$ & 263.61 & 1 & 263.61 & 12.42 & 0.0097 \\
\hline $\mathrm{C}^{2}$ & 617.74 & 1 & 617.74 & 29.09 & 0.0010 \\
\hline Residual & 148.63 & 7 & 21.23 & & \\
\hline Lack of Fit & 61.15 & 3 & 20.38 & 0.93 & 0.5031 \\
\hline Pure Error & 87.48 & 4 & 21.87 & & \\
\hline Cor Total & 3545.04 & 16 & & & \\
\hline \multicolumn{6}{|c|}{$\mathrm{R}^{2}=95.81 \% ; \mathrm{R}_{\mathrm{adj}}^{2}=90.42 \% ;$ Coefficient of variation $(\mathrm{C} . \mathrm{V} ., \%)=6.76$} \\
\hline
\end{tabular}

The trend graphs of the three factors interaction on hydroxyl free radical scavenging rate are given in Fig.

2. The effect of fermentation time on hydroxyl free radical scavenging rate was significant. Moreover, this effect was not only one-dimensional but also quadratic. The hydroxyl free radical scavenging rate increased with the increasing of fermentation time, but the variable trend was relatively uniform. The fermented action might best strengthened and product might increase when fermentation time increased under the condition of fixed solid-to-liquid ratio and inoculum size. Therefore, the hydroxyl free radical scavenging rate of fermented liquid increased. As inoculum size increased, hydroxyl free radical scavenging rate increased with it. However, they cannot beyond the optimum range. More inoculum size would bind to protease active sites so as to strengthen the protease effect and increase the amount of product under the situation of suitable solid-to-liquid ratio. At the optimum reaction temperature, fermented reaction rate reached the maximum value and the maximum fermented product was also obtained. The hydroxyl free radical scavenging rate increased continually with the increase of fermentation time. Under the condition of fixed solid-to-liquid ratio, the more the inoculum size was, the better the effect action of fermentation was and the more the antioxidant product was. 

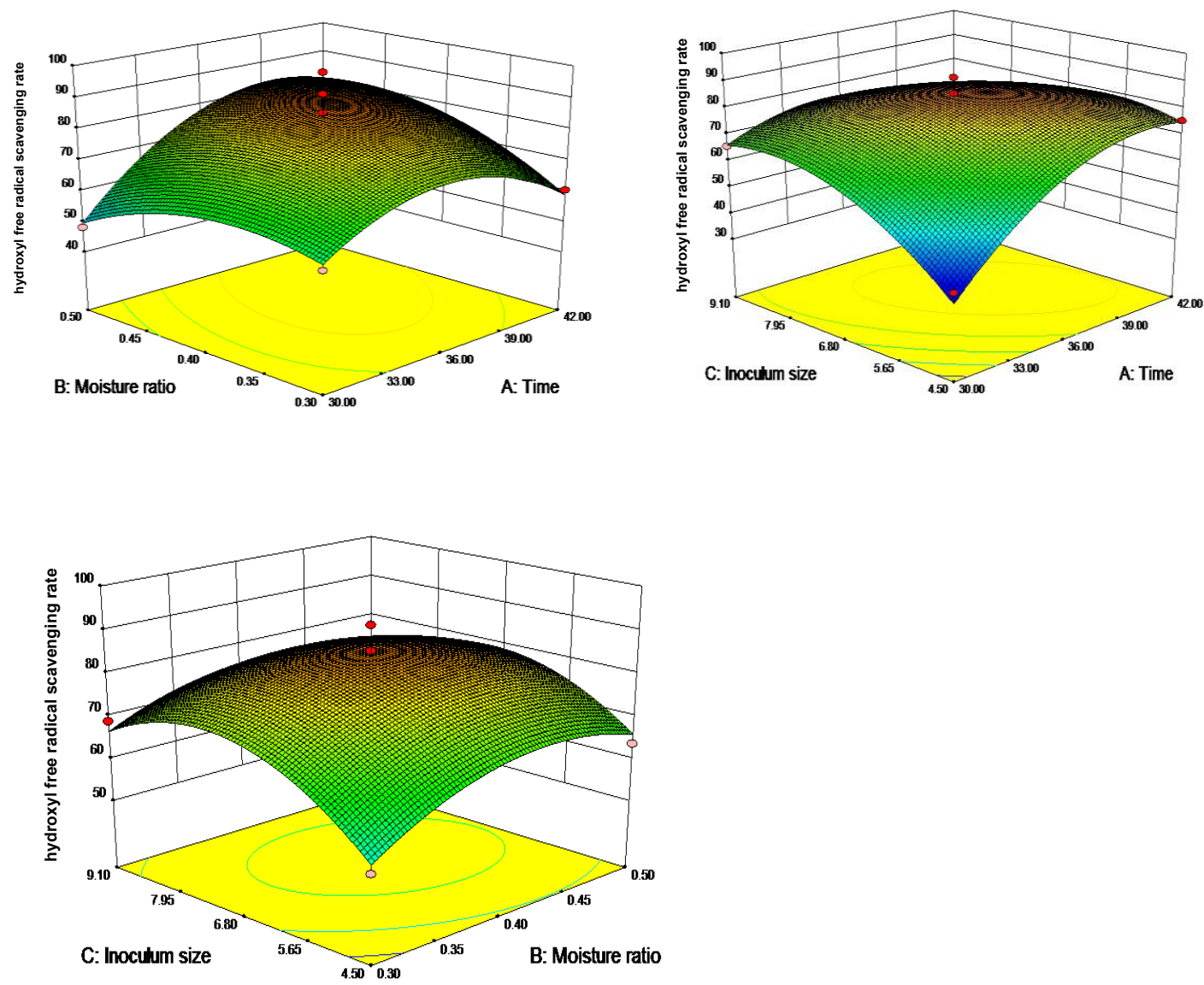

Fig.2 Trend graphs of effect of various factors on fermentation results

The optimum fermented conditions were obtained by using Design-Expert software design and analysis of RSM model as follows: $\mathrm{A}=38.8, \mathrm{~B}=1: 0.44, \mathrm{C}=6.48 \%$, that is $38.8 \mathrm{~h}$ of fermentation time ,material liquid ratiol:0.44, $6.48 \%$ of inoculation amount. The theoretical maximum of hydroxyl free radical scavenging rate is $86.28 \%$.Nevertheless, under the optimum conditions, verifying test result showed that the hydroxyl free radical scavenging rate could reach $87.3 \%$. The relative error of the difference of the result obtained by predicted and actual soluble nitrogen mass concentration is less than $2 \%$. Therefore, result of validate experiment proved that there are good fitness between the predicted and actual value.

\section{Conclusions}

The purpose of this research is to study the optimum fermentation technological condition. According to the single factor experiment and the RSM (BBD) experiment, it was found that the optimum fermentation is: $38.8 \mathrm{~h}$ of Fermentation time, $37^{\circ} \mathrm{C}$ of fermentation temperature, material liquid ratio1:0.44, $6.48 \%$ of inoculation amount. Under this condition, the hydroxy free radical, iron reduction capacity and DPPH radical scavenging rate were $87.3 \%, 0.36$ ( OD value)and $73.4 \%$,respectively.Fermented liquid has effectively antioxidant activity, and using solid state fermentation technology is an effective approach to achieving comprehensive profit of processing peanut meal. Therefore, the in-depth research of the fermentation technology can play very good improvement function on the development of profoundly processed peanut meal protein. 


\section{References}

1. L.N. Yu, Q.L. Yang, S.L. Yu, J. Bi and C.S. Zhang: Science and Technology of Food Industry, (2010), 3,376-380 .

2. Y.H. Zhang and Q. Wang: Transactions of the Chinese Society of Agricultural Engineering, (2007), 23, p. 258

3. Chung, Y. C., Chang, C. T., Chao, W. W., Lin, C. F., \& Chou, S. T. Antioxidant activity and safety of the $50 \%$ ethanolic extract from red bean fermented by Bacillus subtilis IMR-NK1. Journal of Agricultural and Food Chemistry, (2002), 50, 2454-2458.

4. Steinkraus, K. H. Classification of fermented foods: worldwide review of household fermentation techniques. Food Control, (1997) ,8,311-317.

5. Y.P. Zhu, J.F. Fan, Y.Q. Cheng, L.T. Li .Improvement of the antioxidant activity of Chinese traditional fermented okara
(Meitauza) using Bacillus subtilis B2. Food Control, (2008), 19, $654-661$.

6. Amarowicz, R.; Naczk, M.; Shahidi, F. Antioxidant activity of various fractions of non-taninphenolics of canola hulls. J. Agric. Food Chem. (2000), 48, 2755-2759.

7. Khantaphant, S.; Benjakul, S.; Ghomi, M.R. The effects of pretreatments on antioxidative activities of protein hydrolysate from the muscle of browns tripe red snapper (Lutjanusvitta).LWT Food Sci. Technol.( 2011), 44, 1139-1148.

8. You, L.J.; Zhao, M.M.; Cui, C.; Zhao, H.F.; Yang, B. Effect of degree of hydrolysis on the antioxidant activity of loach (Misgurnusanguillicaudatus) protein hydrolysates. Innov.Food Sci. Emerg.(2009), 10, 235-240. 